\title{
The use of CT coronary calcium score in asymptomatic patients with familial hypercholesterolaemia
}

\author{
KATE E SHIPMAN, ${ }^{1}$ ELIZABETH J HIGGINSON, ${ }^{1}$ ANGELA L HADDON, ${ }^{1}$ MIN Y WONG, ${ }^{2}$ \\ MOURAD Z LABIB ${ }^{1}$
}

\begin{abstract}
Background: Familial hypercholesterolaemia $(\mathrm{FH})$ is associated with accelerated atherosclerosis. Diagnosis is based on clinical scores and only $45 \%$ of those with a clinical diagnosis have a genetic mutation known to be associated with FH. Coronary artery calcium (CAC) scoring can be used as a surrogate marker for coronary artery disease, but data on its use in $\mathrm{FH}$ are scarce.

Methods: CAC was performed in $\mathbf{5 2}$ asymptomatic patients (16 'probable FH' and 36 'possible FH') classified according to modified Simon Broome criteria, who were attending a secondary care lipid clinic. Demographic and disease characteristics, CAC (Agatston) scores and arterial age were audited. Results: Half the patients had an Agatston score of zero. In those with Agatston scores $>0$ and age $>45$ years, the mean arterial age was significantly higher than the chronological age ( 72 versus 59 years; $p=0.0001$ ). Only diabetes and hypertension status were significantly different between those with Agatston scores of 0 and $>0(p=0.045$ and $p=0.0175$ respectively). Arterial age provided information useful for individualised clinical management.

Conclusions: Atherosclerosis burden varies widely in $\mathrm{FH}$ with diabetes mellitus and hypertension appearing to contribute. We have found the information from CT calcium scoring very useful in identifying those patients with significant atherosclerosis who would need more intensive treatment.

Br J Diabetes 2016:16:62-64
\end{abstract}

Key words: familial hypercholesterolaemia, CT coronary calcium, Agatston score, atherosclerosis, coronary artery disease

Department of Clinical Biochemistry and Metabolic Medicine, Russells Hall Hospital, Dudley, West Midlands, UK

2 Department of Radiology, Russells Hall Hospital, Dudley, West Midlands, UK

Address for correspondence: Dr Kate Shipman

Department of Clinical Biochemistry, Russells Hall Hospital, Pensnett

Road, Dudley, West Midlands, DY1 2HQ, UK

Tel: +44 (0)1384 244078

E-mail: kate.shipman@doctors.net.uk

http://dx.doi.org/10.15277/bjdvd.2015.039

\section{Introduction}

Familial hypercholesterolaemia (FH) is associated with accelerated atherosclerosis, an increased risk of coronary artery disease (CAD) and premature death. ${ }^{1,2}$ As many as $24 \%$ of asymptomatic patients with $\mathrm{FH}$ have been found to have extensive $C A D$, although $16 \%$ of patients have no CAD. ${ }^{3}$ This heterogeneity and the lack of agreed clinical criteria for the diagnosis of $\mathrm{FH}$ complicates the detection and treatment of $\mathrm{FH}$. The specificity and sensitivity of the diagnostic tools available, such as the UK Simon Broome criteria (National Institute for Health and Care Excellence [NICE]), are not consistent across populations and a genetic mutation consistent with $\mathrm{FH}$ can only be identified in $45 \%$ of those with a clinical diagnosis of this disorder. ${ }^{4}$ Genotyping is not widely available in clinics in England and most clinics, like ours, rely on the Simon Broome clinical classification.

Lifestyle modifications and pharmacotherapy have been shown to significantly delay or prevent the onset of CAD in individuals with $\mathrm{FH}$. In our experience, management is often complicated by poor compliance with lifestyle modifications or medication and statin intolerance, whether true or perceived. Some possible FH cases will present with an atherosclerotic burden (despite having no identified mutation), whereas some definite FH cases will not. ${ }^{2}$ Non-invasive modalities for assessing atherosclerosis, such as carotid ultrasonography and measurement of coronary artery calcium (CAC) scores, have been recommended to stratify asymptomatic FH patients for routine, enhanced and high-intensity treatment. ${ }^{5}$ We have routinely performed CAC in all asymptomatic patients with possible and probable $\mathrm{FH}$ who were attending the secondary care lipid clinic since 2012 and have audited the results.

\section{Patients and methods}

The outcome of CAC in 54 adult patients with a clinical diagnosis of $\mathrm{FH}$ who were attending our secondary care lipid clinic between September 2012 and August 2013 was audited. Patients were classified according to the modified Simon Broome criteria (without genotyping) into 'possible', 'probable' or 'definite' FH (Table 1). ${ }^{5}$ Two patients with 'definite' $\mathrm{FH}$ were excluded from further analysis and 52 patients with 'possible' or 'probable' FH were included in the final analysis. CAC was quantified using multislice CT scanning and patients were classified into four categories according to the Agatston score: 0; 1-100; 101-400; >400. 
Table 1. Modified Simon Broome criteria used for the diagnosis of possible or probable familial hypercholesterolaemia ${ }^{5}$

\begin{tabular}{ll}
\hline Point & Criteria \\
A & DNA mutation \\
B & Tendon xanthomas in patient or 1 st or 2 nd-degree relative \\
C & Family history of myocardial infarction $<50$ years in 2 nd-degree or \\
& $<60$ years in $1 \mathrm{st}$-degree relative \\
D & Family history of total cholesterol $>7.5 \mathrm{mmol} / \mathrm{L}$ in $1 \mathrm{st} / 2$ nd-degree relative \\
E & Total cholesterol $>7.5 \mathrm{mmol} / \mathrm{L}$ (adult) or $>6.7 \mathrm{mmol} / \mathrm{L}$ (age $<16$ years) \\
$\mathrm{F}$ & $\mathrm{LDL}$-cholesterol $>4.9 \mathrm{mmol} / \mathrm{L}$ (adult) or $>4.0 \mathrm{mmol} / \mathrm{L}$ (age $<16$ years) \\
Definite $\mathrm{FH}:$ Hypercholesterolaemia as defined in points $\mathrm{E} / \mathrm{F}$ plus $\mathrm{A}$ \\
Probable $\mathrm{FH}:$ Hypercholesterolaemia as defined in points E/F plus B \\
Possible FH: Hypercholesterolaemia as defined in points E/F plus either C or D
\end{tabular}

Data on age, gender, serum cholesterol, $\mathrm{HbA}_{1 c}$, prevalence of diabetes, hypertension and vascular disease, smoking status and length of time on statin prior to performing CAC were collected from patient notes and laboratory results. Normally distributed data are presented as mean \pm SD and non-normally distributed data as median and IQR. Appropriate statistical tests were performed, including unpaired and paired t-tests, MannWhitney $U$ test, Pearson-chi squared and Wilcoxon rank sum (with continuity correction) tests, using Microsoft Office Excel ${ }^{\circledR}$ 2003, Analyse-it ${ }^{\circledR}$ (Version 2.26) and R (Version 3.1.26); $p<0.05$ was considered statistically significant. ${ }^{6}$ The Multi-Ethnic study of Atherosclerosis (MESA) 'Arterial Age' online calculator was used to calculate the heart age of those aged $>45$ years (estimated age of a person with arteries with the same calcium burden). ${ }^{7,8}$

\section{Results}

Demographic and clinical data for the 52 patients with a clinical diagnosis of $\mathrm{FH}$ are listed in Table 2; $6 \%$ had diabetes and 29\% were current smokers. Half had an Agatston score of 0 , about onequarter had scores 1-100, with lower proportions in the higher CAC categories. For 17 patients with Agatston scores $>0$ and older than 45 years, the mean heart age was 72 years which was significantly higher than their chronological age of 59 years $(p=0.0001)$. Only three patients in total had a past medical history of any vascular disease (Agatston scores 0, 135.5 and 187). There were no significant differences in age, sex, smoking status or length of time on statin prior to the CT scan between patients with Agatston scores of 0 or $>0$, although significantly more patients with diabetes or hypertension were in the higher Agatston score category (Table 3). Indeed, no patient with diabetes had an Agatston score of 0 and 9/11 patients with hypertension had high Agatston scores (Table 3). The median (IQR) Agatston score was significantly higher for the 13 patients with diabetes and/or hypertension versus those without either (136 [26-381] versus 0 [0-50]), respectively; $p=0.003)$. This difference remained significant if current smokers were included within the group to give 24 patients who either smoked, had diabetes and/or hypertension (58 [0-200] versus 0 [0-14]) respectively; $p=0.003$ ).
Table 2. Demographic, biochemical and clinical data of 52 subjects with possible and probable familial hypercholesterolaemia

\begin{tabular}{ll}
\hline Demographic characteristics & \\
Male / female & $19(37) / 33(10.9 \pm 10.9$ \\
Age (years) & \\
\hline Metabolic parameters & \\
Total cholesterol (mmol/L) & $9.2 \pm 1.3$ \\
LDL-cholesterol (mmol/L) & $6.5 \pm 1.5$ \\
Triglycerides (mmo//L) & $2.0 \pm 1.0$ \\
HDL-cholesterol (mmol/L) & $1.6 \pm 0.4$ \\
HbA $_{1 C}(\%)$ & $5.7 \pm 0.5$
\end{tabular}

Comorbidities and cardiovascular risk factors

$\begin{array}{ll}\text { Diabetes } & 3(6) \\ \text { Current smokers } & 15(29) \\ \text { Hypertension } & 11(21) \\ \text { Prior medical history of vascular disease } & 3(6) \\ \text { Median Agatston score (IQR, max) } & 1.8(0-72,700) \\ \text { Distribution of Agatston scores } & \\ 0 & 26(50) \\ 1-99 & 14(27) \\ 100-399 & 8(15) \\ \geq 400 & 4(8)\end{array}$

Data are $\mathrm{N}(\%)$ or means \pm SD, except where indicated. Diagnosis was according to modified Simon Broome criteria (see text and Table 1). IQR: interquartile range.

Table 3. Demographic and disease characteristics stratified by Agatston scores

\begin{tabular}{llll}
\hline & \multicolumn{2}{l}{ Agatston score } & p \\
& $\mathbf{0}$ & $\geq \mathbf{1}$ & \\
Number & 26 & 26 & \\
Age (years) & $49 \pm 11$ & $52 \pm 11$ & 0.2787 \\
Male (n) & 7 & 12 & $0.1499^{\mathrm{a}}$ \\
\hline Diabetes (n) & 0 & 3 & $0.045 \mathrm{a}$ \\
Hypertension (n) & 2 & 9 & $0.0175^{\mathrm{a}}$ \\
Smoking (n) & 5 & 10 & \\
& $(4$ ex-smokers) & $(3$ ex-smokers) & $0.105^{\mathrm{a}}$ \\
\hline $\begin{array}{l}\text { Months on statin prior to scan } \\
\text { [median (IQR)] }\end{array}$ & 7 (5-36) & 17 (6-142) & $0.1393^{\mathrm{b}}$
\end{tabular}

$\mathrm{p}$ Values calculated by t-test, or aPearson-chi squared test, or bMann Whitney U Test. IQR: interquartile range.

\section{Discussion}

The burden of atherosclerosis varies widely among people with possible or probable $\mathrm{FH},{ }^{3}$ with the median CAC score being lower than the mean reported for a cohort of 50 definite $\mathrm{FH}$ patients in a study from Spain. ${ }^{9}$ Although high-intensity statin therapy is recommended for all patients with $\mathrm{FH},{ }^{10}$ in our experience, not all respond equally and some cannot tolerate this treatment. We have found that the addition of information from CAC score is useful in deciding the intensity of treatment and aids compliance in asymptomatic 


\section{Key messages}

- Agatston scores varied widely in asymptomatic patients with possible and probable familial hypercholesterolaemia

- Among other risk factors of cardiovascular disease, only diabetes mellitus and hypertension were associated with higher coronary calcium scores

- Arterial age was useful in guiding intensity of statin treatment and in improving compliance

individuals. For example, one patient, a newly-diagnosed 66 year old woman, with an Agatston score of zero, and at a low risk of an imminent CAD event (arterial age 39 years), was kept at a $20 \mathrm{mg}$ dose as she had side effects at higher doses. Conversely, a 52 year old man who was reluctant to start a statin became immediately keen to start high-intensity treatment when the CAC score was 72 , giving him a heart age of 70 years. 7,8

While the Simon Broome criteria are useful for identifying people likely to have a genetic dyslipidaemia, the classification itself does not accurately reflect the burden of atherosclerosis and intensity of treatment. ${ }^{3}$ Conventional cardiovascular risk factors, such as diabetes, hypertension and smoking, appear to contribute to the overall atherosclerotic burden, as would be expected. Our audit showed that, in those with a clinical diagnosis of $\mathrm{FH}$, the added information of CAC was highly valuable for deciding on the intensity of treatment and for supporting compliance with treatment.

The small patient population is a limitation of this study. We will continue to audit clinic data but have also been fortunate enough to be invited to collaborate with another group, led by Professor S Humphries, who are similarly auditing the results of their innovative clinic pathway. This group performs CAC scans as well as genotyping and, by combining datasets, firmer conclusions may be drawn. Continued work in this area will then define the utility of CAD risk assessment by CAC scoring in people with $\mathrm{FH}$. Only then will we be in a position to assess the contribution of this technique to patient care and outcomes.

\section{Conclusions}

The burden of atherosclerosis varied widely among patients with possible and probable $\mathrm{FH}$, as observed previously. The presence of other well-established cardiovascular risk factors, such as diabetes mellitus and hypertension, may also contribute to the burden of atherosclerosis in these patients. We have found the information from $\mathrm{CT}$ calcium scoring very useful in identifying those patients with significant atherosclerosis who would likely benefit from more intensive treatment.

Conflict of interest All authors declare there are no conflicts of interest.

Funding None received.

Acknowledgments We would like to thank the radiology and cardiology departments of Russells Hall Hospital for their support in providing this service to the lipid clinic as well as the staff of the lipid clinic for their ongoing work on behalf of the lipid patients. We would like to mention $\mathrm{Dr}$ $\mathrm{M}$ Wong specifically for her help in interpretation of scan reports and her dedication to providing this important service to the patients.

\section{References}

1. Austin M, Hutter CM, Zimmern RL, et al. Familial hypercholesterolaemia and CHD: a HuGE association review. Am J Epidemiol 2004;160:421429.

2. Neefjes L, Ten Kate GJ, Rossi A, et al. CT coronary plaque burden in asymptomatic patients with familial hypercholesterolaemia. Heart 2011;97(14):1151-7. http://dx.doi.org/10.1136/hrt.2010.220699

3. Neefjes L, Ten Kate GJ, Alexia R, et al. Accelerated subclinical coronary atherosclerosis in patients with familial hypercholesterolaemia. Atherosclerosis 2011;219(2):721-7. http://dx.doi.org/10.1016/j.atherosclerosis.2011.09.052

4. Ho CK, Stirling D, Hannant W, et al. Genetic mutations in SE Scotland Scott Med J 2012;57(3)148-151. http://dx.doi.org/10.1258/smj.2012.012020

5. The Cardiac Society of Australia and New Zealand. CSANZ guidelines for the diagnosis and management of familial hypercholesterolaemia. http://www.csanz.edu.au/wp-content/uploads/2013/12/Familial_Hypercholesterolemia_2013.pdf. 2010 (accessed 8 August 2014).

6. R Core Team. R: A language and environment for statistical computing. R Foundation for Statistical Computing, Vienna, Austria. http://www.Rproject.org/. 2014 (accessed 1 May 2015).

7. McClelland RL, Nasir K, Budoff M, et al. Arterial age as a function of coronary artery calcium (from the Multi-Ethnic study of Atherosclerosis [MESA]). Am J Cardiol 2009;103(1):59-63.

http://dx.doi.org/10.1016/j.amjcard.2008.08.031

8. The Multi-Ethnic Study of Atherosclerosis. http://www.mesanhlbi.org/Calcium/ArterialAge.aspx. (accessed 10 January 2015)

9. Viladés Medel D, Leta Petracca R, Carreras Costa F, et al. Coronary computed tomographic angiographic findings in asymptomatic patients with heterozygous familial hypercholesterolemia and null allele low-density lipoprotein receptor mutations. Am J Cardiol 2013;111(7):955-61. http://dx.doi.org/10.1016/j.amjcard.2012.12.012

10. National Institute for Health and Care Excellence (NICE) guidelines [CG71]. Identification and management of familial hypercholesterolaemia. http://www.nice.org.uk/Guidance/CG71. August 2008 (accessed 9 November 2014). 